\title{
ITER test blanket module error field simulation experiments at DIII-D
}

\author{
M.J. Schaffer ${ }^{1}$, J.A. Snipes ${ }^{2}$, P. Gohil ${ }^{1}$, P. de Vries $^{3}$, T.E. Evans ${ }^{1}$, M.E. Fenstermacher ${ }^{4}$, \\ X. Gao ${ }^{5}$, A.M. Garofalo ${ }^{1}$, D.A. Gates ${ }^{6}$, C.M. Greenfield ${ }^{1}$, W.W. Heidbrink ${ }^{7}$, \\ G.J. Kramer ${ }^{6}$, R.J. La Haye ${ }^{1}$, S. Liu ${ }^{5}$, A. Loarte ${ }^{2}$, M.F.F. Nave ${ }^{8}$, T.H. Osborne ${ }^{1}$, \\ N. Oyama ${ }^{9}$, J.-K. Park $^{6}$, N. Ramasubramanian ${ }^{10}$, H. Reimerdes $^{11}$, G. Saibene ${ }^{12}$, \\ A. Salmi ${ }^{13}$, K. Shinohara ${ }^{9}$, D.A. Spong ${ }^{14}$, W.M. Solomon ${ }^{6}$, T. Tala ${ }^{15}$, Y.B. Zhu ${ }^{7}$, \\ J.A. Boedo ${ }^{16}$, V. Chuyanov ${ }^{2}$, E.J. Doyle ${ }^{17}$, M. Jakubowski ${ }^{18}$, H. Jhang ${ }^{19}$, R.M. Nazikian ${ }^{6}$, \\ V.D. Pustovitov ${ }^{20}$, O. Schmitz ${ }^{21}$, R. Srinivasan ${ }^{10}$, T.S. Taylor ${ }^{1}$, M.R. Wade ${ }^{1}$, K.-I. You ${ }^{19}$, \\ L. Zeng ${ }^{17}$, and the DIII-D Team
}

${ }^{1}$ General Atomics, PO Box 85608, San Diego, CA 92186-5608, USA

${ }^{2}$ ITER Organization, Route de Vinon sur Verdon, F-13115 St Paul Lez Durance, France

${ }^{3}$ FOM Institute for Plasma Physics Rijnhuizen, Association EURATOM-FOM, 3430 BE Nieuwegein, Netherlands

${ }^{4}$ Lawrence Livermore National Laboratory, Livermore, CA 94550, USA

${ }^{5}$ ASIPP, Hefei, Anhui 230031, People's Republic of China

${ }^{6}$ Princeton Plasma Physics Laboratory, PO Box 451, Princeton, NJ 08543, USA

${ }^{7}$ University of California-Irvine, Irvine, CA 92697, USA

${ }^{8}$ Associação EURATOM/IST, Instituto de Plasmas e Fusão Nuclear, Lisbon, Portugal

9 JAEA, 801-1, Mukouyama, Naka City, Ibaraki, 311-0193, Japan

${ }^{10}$ Institute for Plasma Research, Bhat, Gandhinagar, India

${ }^{11}$ Columbia University, New York, NY 10027, USA

${ }^{12}$ Fusion for Energy Joint Undertaking, Josep Pla. 2, 08019 Barcelona, Spain

${ }^{13}$ Association EURATOM-Tekes, Aalto University, FI-00076, AALTO, Finland

${ }^{14}$ Oak Ridge National Laboratory, PO Box 2008, Oak Ridge, TN 37831, USA

${ }^{15}$ Association EURATOM-Tekes, VTT, FI-02044 VTT, Finland

${ }^{16}$ University of California-San Diego, San Diego, CA 92093-0417, USA

${ }^{17}$ University of California-Los Angeles, Los Angeles, CA 90095, USA

${ }^{18}$ Max-Planck-Institut für Plasmaphysik, Association EURATOM-MPI, Greifswald, Germany

${ }^{19}$ National Fusion Research Institute, Daejeon 305-333, Korea

${ }^{20}$ Institute of Tokamak Physics, Russian Research Centre Kurchatov Institute, Moscow, Russian Federation

${ }^{21}$ FZ Jülich, IEF4-Plasma Physics, Association EURATOM-FZJ, 52428 Jülich, Germany

Received 8 February 2011, accepted for publication 10 August 2011

Published 2 September 2011

Online at stacks.iop.org/NF/51/103028

\begin{abstract}
Experiments at DIII-D investigated the effects of magnetic error fields similar to those expected from proposed ITER test blanket modules (TBMs) containing ferromagnetic material. Studied were effects on: plasma rotation and locking, confinement, L-H transition, the H-mode pedestal, edge localized modes (ELMs) and ELM suppression by resonant magnetic perturbations, energetic particle losses, and more. The experiments used a purpose-built three-coil mock-up of two magnetized ITER TBMs in one ITER equatorial port. The largest effect was a reduction in plasma toroidal rotation velocity $v$ across the entire radial profile by as much as $\Delta v / v \sim 60 \%$ via non-resonant braking. Changes to global $\Delta n / n, \Delta \beta / \beta$ and $\Delta \mathrm{H}_{98} / \mathrm{H}_{98}$ were $\sim 3$ times smaller. These effects are stronger at higher $\beta$. Other effects were smaller. The TBM field increased sensitivity to locking by an applied known $n=1$ test field in both L- and H-mode plasmas. Locked mode tolerance was completely restored in L-mode by re-adjusting the DIII-D $n=1$ error field compensation system. Numerical modelling by IPEC reproduces the rotation braking and locking semi-quantitatively, and identifies plasma amplification of a few $n=1$ Fourier harmonics as the main cause of braking. IPEC predicts that TBM braking in H-mode may be reduced by $n=1$ control. Although extrapolation from DIII-D to ITER is still an open issue, these experiments suggest that a TBM-like error field will produce only a few potentially troublesome problems, and that they might be made acceptably small.
\end{abstract}

(Some figures in this article are in colour only in the electronic version) 


\section{Introduction}

The proposed ITER tritium-breeding test blanket modules (TBMs) [1] are each expected to contain about 1 tonne of high-temperature and neutron tolerant martensitic steel. The contemplated steel alloys are ferromagnetic and will perturb the nearby plasma with $\sim 1 \%$ local magnetic field reductions [2] in addition to the usual toroidal field (TF) coil ripple. Serious deleterious effects, especially on H-mode performance, were feared based on past experience with toroidal field ripple from discrete TF coils on the large JT-60U and JET tokamak plasmas [3,4]. However, whereas TF coil ripple is periodic, the TBM field consists of a few localized aperiodic magnetic 'bumps'. The planned ITER installation consists of six TBMs of varied designs from the ITER parties. They will be distributed two per port in each of three large equatorial ports, spaced $40^{\circ}$ apart toroidally. Ferromagnetic steel in the modules concentrates magnetic flux, thereby weakening the magnetic field at the plasma between TF coils. TBM perturbations add to and deepen the ripple depth beyond that of the TF coils alone; see figure 1. Because the TBM field lacks a simple symmetry about the torus, it contains $n=1$ harmonics ( $n=$ number of toroidal periods). $n=1$ harmonics are of special concern, because some of them couple strongly to $n=1$ tokamak MHD modes and are associated with plasma rotation braking, locked modes and the formation of large magnetic islands with serious loss of plasma confinement.

It is important to understand the nature of magnetic perturbations produced by small (relative to the plasma) ferromagnetic objects close to the plasma boundary, such as a TBM. The ferromagnetic material is magnetized predominantly in the direction of the strong tokamak axisymmetric field $\boldsymbol{B}_{0}=\boldsymbol{B}_{\mathrm{T} 0}+\boldsymbol{B}_{\mathrm{P} 0}$ encountered just outside of the plasma. Figure 2 shows the pattern of the normal component $B_{r}$ of the TBM magnetic field into and out of an ITER plasma on a magnetic surface near the separatrix, at normalized poloidal flux $\psi_{\mathrm{N}}=0.9975$, computed from a reduced model of the magnetized TBM steel. It is apparent that the $B_{r}$ pattern also gets partly oriented in the $\boldsymbol{B}_{0}$ direction. A magnetic line in the plasma, that passes near the TBM, is deflected almost equally outwards and inwards by the TBM $B_{r}$, due to the short distance along the line over which $B_{r}$ acts [5]. Magnetic line tracing verifies that almost no net radial displacement of the line results from passing near the TBM. This is in contrast to a resonant magnetic perturbation (RMP), which gives more displacement in one radial direction than the other when integrated along initially closed magnetic lines on a rational magnetic surface (resonance). RMPs make magnetic islands in toroidal magnetic field systems. The TBM perturbations are almost entirely non-resonant in this sense, so only small magnetic islands may be expected from them. The inherent smallness of resonant harmonics in the TBM field is seen in the $n=1$ Fourier harmonic spectrum in figure 3, where the magnetic resonance condition, $m=n q$, lies in a spectral valley. Here $m$ is the number of poloidal periods of a helical harmonic and $q$ is the magnetic stability safety factor. These are general properties of the perturbation field of any small, soft or saturated ferromagnetic object immersed in the near-plasma magnetic field of a tokamak. However, the spatially localized

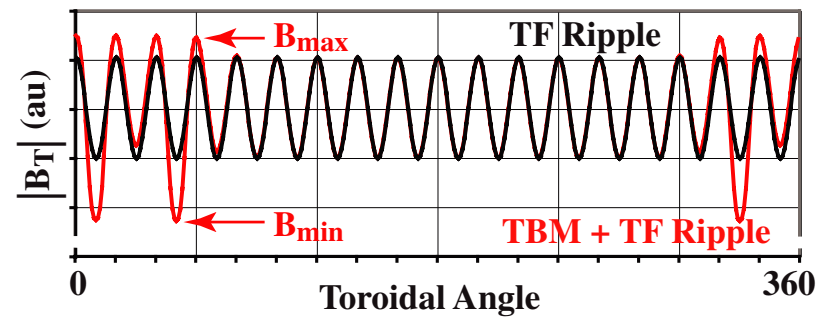

Figure 1. Qualitative plot of the variation of $\left|B_{\mathrm{T}}\right|$ around the equatorial circumference of the ITER plasma at major radius $R=8 \mathrm{~m}$. The black curve represents the ripple of 18 ITER TF coils located at toroidal angles $0^{\circ}, 20^{\circ}$, etc. The red/grey curve shows the combined variation when TBMs are introduced into equatorial ports at toroidal angles $-30^{\circ}, 10^{\circ}$ and $50^{\circ}$.

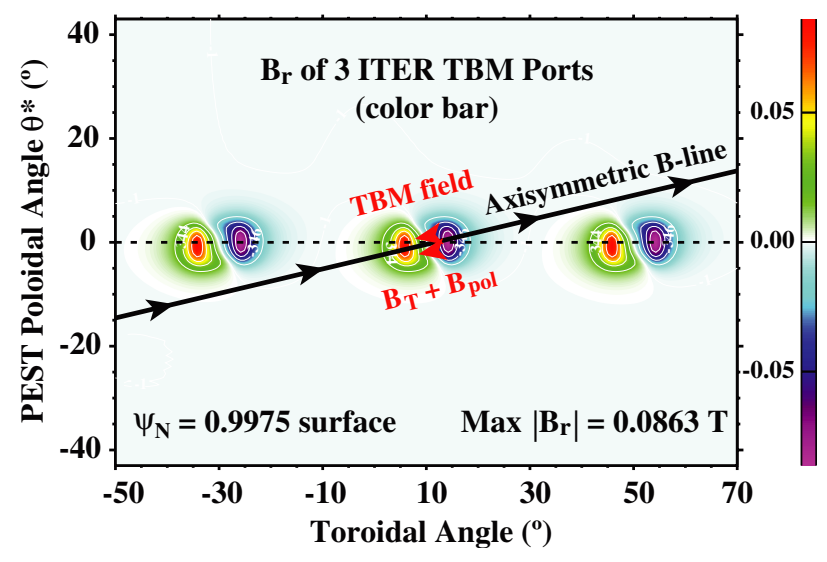

Figure 2. Contour plot of $B_{r}(\mathrm{~T})$, the normal component of the TBM magnetic field, on a magnetic surface near the separatrix. The PEST poloidal angle on an axisymmetric toroidal surface obeys $\mathrm{d} \theta^{*}=q^{-1} \mathrm{~d} \phi$ where $\phi$ is the toroidal angle, which makes magnetic lines appear as straight lines. The TBM steel (not shown, but located slightly 'above' the plotted surface) is magnetized in the direction of the strong axisymmetric field. The magnetization drives a magnetic flux out to the right, some of which enters the plasma (blue and violet contours) and returns through the plasma (short red vectors) and out (green-yellow-red contours) back into the steel.

TBM perturbation generates hundreds of non-resonant helical Fourier harmonics, which are strongest for $7 \leqslant n \leqslant 30$.

Many of the effects of TBM perturbations on ITER plasmas cannot be predicted completely with confidence from present theory. Therefore, at the request of the ITER Organization (IO), a TBM error field mock-up was designed and temporarily installed in an equatorial port at DIII-D to assess TBM effects experimentally. The experiments were planned and performed in 2009 by DIII-D staff and collaborators, plus a specially nominated international team of scientists. Specific results have been presented at conferences in 2010 [6-8]. This paper amplifies those earlier papers and provides further information about the experiments and their interpretation.

\section{TBM error mock-up at DIII-D}

One large DIII-D equatorial port was temporarily made available to mock up the magnetization of two ITER TBMs installed in one ITER equatorial port. The ITER and DIII-D magnetic geometries are very similar near the plasma, and 


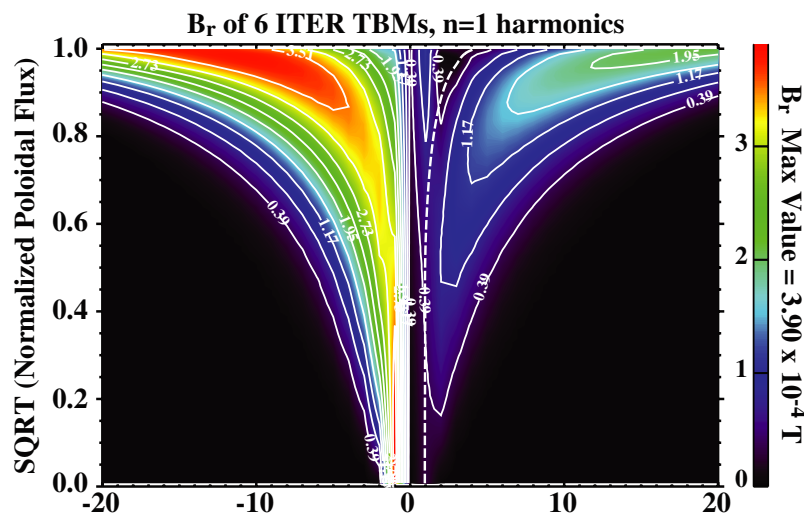

Poloidal Mode Number (m), Neg $\mathbf{m}$ are Left, Pos $\mathbf{m}$ are Right-Handed

Figure 3. Contour plot of $n=1$ Fourier helical harmonic amplitudes of $B_{r}$, the normal component of the TBM magnetic field. The horizontal axis is poloidal harmonic $m$. Negative and positive $m$ are left and right handed, respectively. The vertical axis is the minor radius coordinate $\sqrt{\psi_{\mathrm{N}}}$. The dashed curve marks the condition for magnetic resonance, $m=n q$.

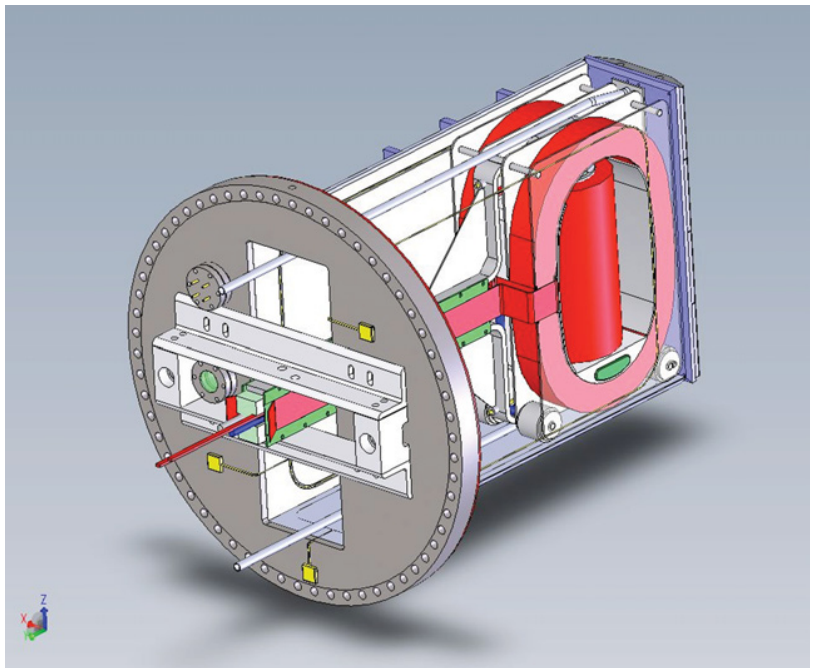

Figure 4. Drawing of TBM mock-up, showing the two racetrack coils and the vertical solenoid coil between them. The coil frame rolls radially on small wheels inside a rectangular enclosure and vacuum barrier. Graphite tiles cover the plasma-facing end.

they are related by a geometric scale factor of about 3.55 . Copper coils were chosen instead of ferromagnetic steel for the TBM error mock-up, because active coils allow much more comprehensive experiments to be performed. However, coils do not respond to changing plasma fields in the same way as ferromagnetic material [9].

The magnetization $M_{\mathrm{T}}$ of the steel of two ITER TBMs in the ITER toroidal magnetic field was simulated by two sideby-side 'racetrack' shaped main coils, visible in figure 4 . The mock-up also contained a vertical solenoid to approximate the poloidally directed magnetization $M_{\mathrm{P}}$ of two ITER TBMs. $M_{\mathrm{P}}$ was included to reproduce the very low resonant harmonic content of the TBM error field already discussed in section 1 . The racetrack and solenoid coils were separately powered by preprogrammed, regulated power supplies, usually to produce 1 or 2 square mock-up current pulses during the tokamak plasma flattop interval. The current directions in the mock-
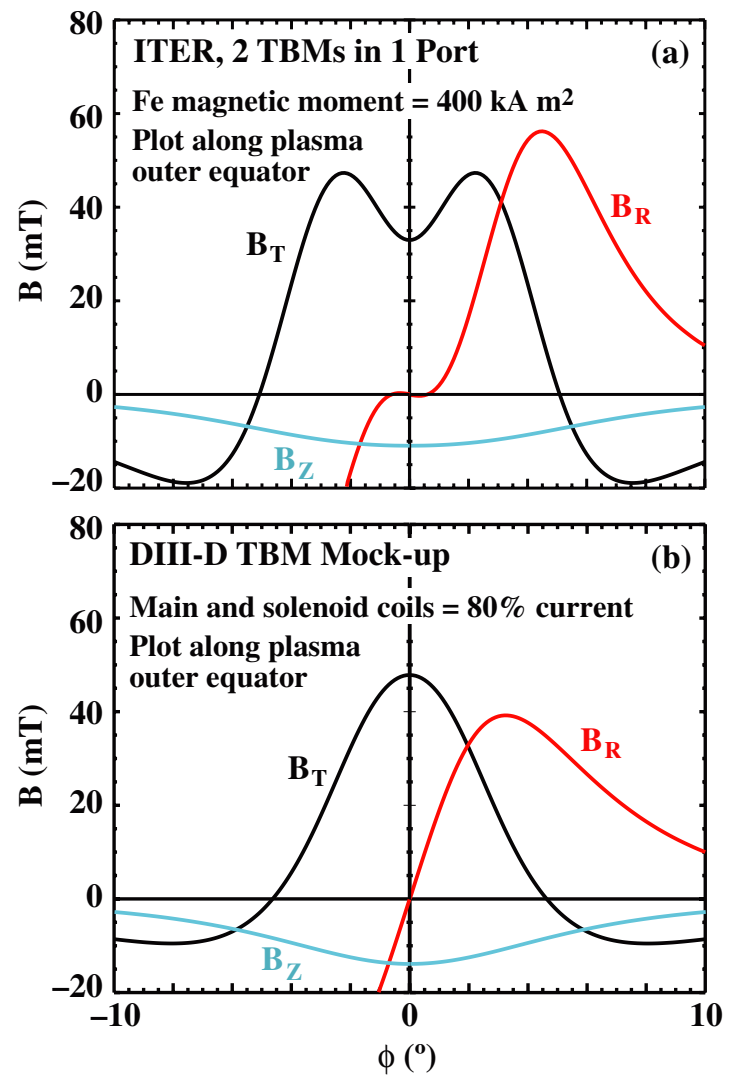

Figure 5. Comparison of the radial, toroidal and vertical $(Z)$ magnetic field components of $(a)$ the ITER TBM and $(b)$ the DIII-D mock-up at their respective plasma outer equatorial major radii. The horizontal axis is the toroidal angle measured from the toroidal centre of the port. Signs of corresponding field components between ITER and DIII-D are made the same here for ease of comparison. Actual perturbation signs depend on the direction of the tokamak $B_{0}$ that magnetizes the steel.

up coils were always set to correspond to the correct direction for steel magnetization in the DIII-D magnetic field, and in most experiments the ratio of the coil currents was adjusted to align the mock-up 'magnetization' parallel with the local tokamak $B_{0}$.

The mock-up coil geometries were chosen to approximate the perturbation field geometry from the plasma surface to the magnetic axis as computed for a preliminary design of two side-by-side ITER TBMs at saturation magnetization [2]. The racetrack coil copper conductors extended $0.45 \mathrm{~m}$ vertically, $0.16 \mathrm{~m}$ toroidally and $0.31 \mathrm{~m}$ radially. Compared with the dimensions of the ITER TBM ferromagnetic structure divided by a 3.55 scale factor, the mock-up racetrack coil assembly was almost exactly the correct height, but it was only half as wide toroidally as the two scaled TBMs. Finally, the mockup racetrack coils were made about 2 times wider radially than the scaled TBM steel, to partly correct the $B_{R}$ (major radius directed) near-field geometry that was distorted due to the small mock-up toroidal width. Figure 5 compares the TBM and mock-up fields at the respective plasma equators. The TBM near field at the ITER plasma equator clearly shows the presence of the two separated modules, whereas the proportionately narrower mock-up field at the DIII-D equator does not. The mock-up $B_{\mathrm{T}}$ perturbation decays at the rate of 
about $10 \%$ per radial centimetre in the neighbourhood of the plasma boundary.

Because ITER will have three TBM ports while DIII-D has a mock-up in only one port, the mock-up was specified to be capable of at least 3 times the normalized perturbation, $\left[\delta B / B_{0}\right]_{\text {ITER }}$ of one ITER TBM port. Since the ratio of ITER to DIII-D central toroidal fields is $(5.3 \mathrm{~T}) /(2.14 \mathrm{~T}) \approx 2.5$, it follows that the absolute $[\delta B]_{\text {DIII-D }}$ capability of the mock-up was slightly larger than the absolute $[\delta B]_{\text {ITER }}$ from an ITER TBM port. We do not claim that $\left[\delta B / B_{0}\right]_{\text {DIII-D }}=3 \cdot\left[\delta B / B_{0}\right]_{\mathrm{ITER}}$ is a proper scaling of experimental results from DIII-D to ITER, and the subject of scaling will be addressed again briefly in section 5 of this paper.

For most experiments, the mock-up coil set was inserted to the end of its rectangular enclosure, its design position. However, it could be rolled radially and secured in 12 equally spaced positions within the enclosure, in order to test the effects of TBM 'recession' by up to an additional $1 \mathrm{~m}$ away from the ITER plasma. This corresponded to a designed $0.28 \mathrm{~m}$ radial range of the mock-up positions in DIII-D.

\section{Experimental procedure}

A list of experimental questions of concern to the IO and DIII-D organizations was assembled and prioritized in discussions beforehand between the two entities. Because there was no prior experience with a TBM-like error, the first two days of DIII-D experiments were dedicated to a 'survey' of the listed topics and development of suitable test plasmas. It was quickly discovered that the mock-up field at the level of one ITER TBM port elicited little or no measureable response from most plasmas tested. Therefore, most of the survey was performed at full mock-up field intensity so as to maximize the chances to identify TBM effects. After the survey, the main campaign was organized to investigate in more detail those high-priority topics that had shown evidence of sensitivity to the mock-up field.

Most experiments of the main campaign were executed in lower-single-null diverted, pumped H-mode plasmas with frequent regular type I edge localized modes (ELMs). The DIII-D intrinsic error field was compensated for by a standard correction scheme (in use since year 2006) that uses the well-characterized DIII-D 'I-coil' array [10], connected with a $240^{\circ}$ toroidal phase difference between $n=1$ current distributions in the upper and lower rows of the array. The field from this connection couples well to $n=1$ modes in the plasma [11]. However, higher- $n$ intrinsic error harmonics were not compensated in any way. All plasmas used deuterium as the main ion species. Carbon, from the graphite-tiled divertor target and walls, was the main impurity element. Neutral beam heating, when used, was always by deuterium beams. The plasma magnetic geometry was an approximate ITER-similar shape (ISS), such as shot 140149 at time $t=$ $2100 \mathrm{~ms}$ shown in figure 6 . Figure 6 also shows the mockup racetrack coil and its location relative to the plasma when fully inserted into its enclosure. The plasma equatorial major radius $R_{\text {midout }}$ was $2.30 \pm 0.004 \mathrm{~m}$ (versus $8.2 \mathrm{~m}$ for ITER). This outer radius was selected for the TBM experiments, because it is where the toroidal field coil periodic ripple from the 24 DIII-D TF coil bundles is about $0.35 \%$, to match

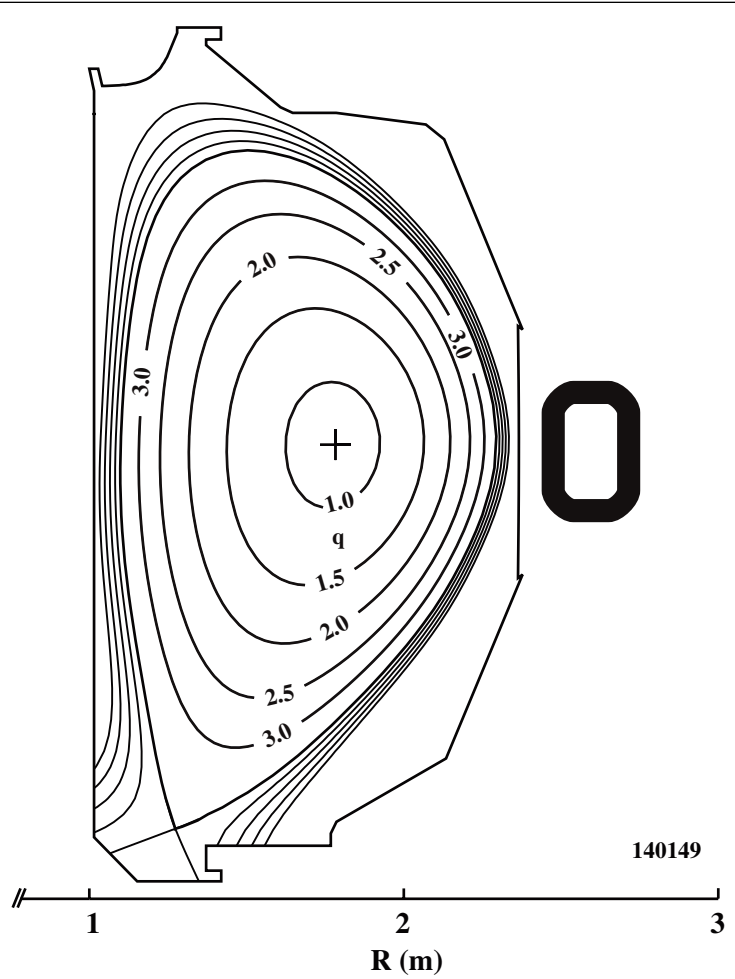

Figure 6. Cross section of DIII-D plasma, vacuum vessel inner surface, and TBM mock-up racetrack coil. Shot 140149 at $t=2100 \mathrm{~ms}$ had the 'ISS' with $R_{\text {midout }} \approx 2.300 \mathrm{~m}$, used in most of these experiments. The coil and its distance from the plasma when fully inserted into its enclosure are approximately to scale.

the ITER goal of $0.35-0.4 \%$ periodic ripple from $18 \mathrm{TF}$ coils after ripple correction by ferromagnetic inserts [2]. For $R_{\text {midout }} \leqslant 2.32 \mathrm{~m}$ the DIII-D plasma performance was still only weakly influenced by proximity to the graphite-tiled DIIID wall, but at $R_{\text {midout }}=2.33 \mathrm{~m}$ the wall interaction was notable, and no further experiments were performed at $2.33 \mathrm{~m}$ and beyond. Other parameters of shot 140149 were $R_{0}=$ $1.70 \mathrm{~m}, a=0.60 \mathrm{~m}$, elongation $=1.80$, upper and lower triangularity $=0.32$ and 0.71 , respectively, $B_{\mathrm{T} 0}=1.72 \mathrm{~T}$, $I_{\mathrm{P}}=1.41 \mathrm{MA}, q_{95}=3.45, q_{0} \approx 0.9$, stored energy $\approx 1 \mathrm{MJ}$, $\beta_{\mathrm{T}} \approx 3 \%, \beta_{\mathrm{P}} \approx 80 \%, \beta_{\mathrm{N}} \approx 2.1, l_{\mathrm{i}} \approx 0.75, l_{\mathrm{i} 3} \approx 0.58$, electron and ion collisionalities $0.1-0.2$ at the top of the $\mathrm{H}$ mode profile pedestal. Other ELMy H-mode experiments used $3.1 \leqslant q_{95} \leqslant 4.3$ and $1.5 \leqslant \beta_{\mathrm{N}} \leqslant 2.5$.

Differences from shot 140149 will be described when the corresponding experiments are presented.

In this paper we characterize the magnitude of the non-axisymmetric perturbing field by a single local ripple parameter,

$$
\delta=\left(B_{\max }-B_{\min }\right) /\left(B_{\max }+B_{\min }\right),
$$

where $B_{\max }$ and $B_{\min }$, identified in figure 1, are the maximum and minimum values of the total toroidal magnetic field $B_{\mathrm{T}}$ at the plasma separatrix in front of the TBM due to the combined TBM and TF coil non-axisymmetries. This is an extension of the usual definition used for periodic, but non-sinusoidal, TF ripple. Since most experiments were performed with the full mock-up field applied, in order to produce larger, easier to measure plasma responses, our local combined $\delta$ was 

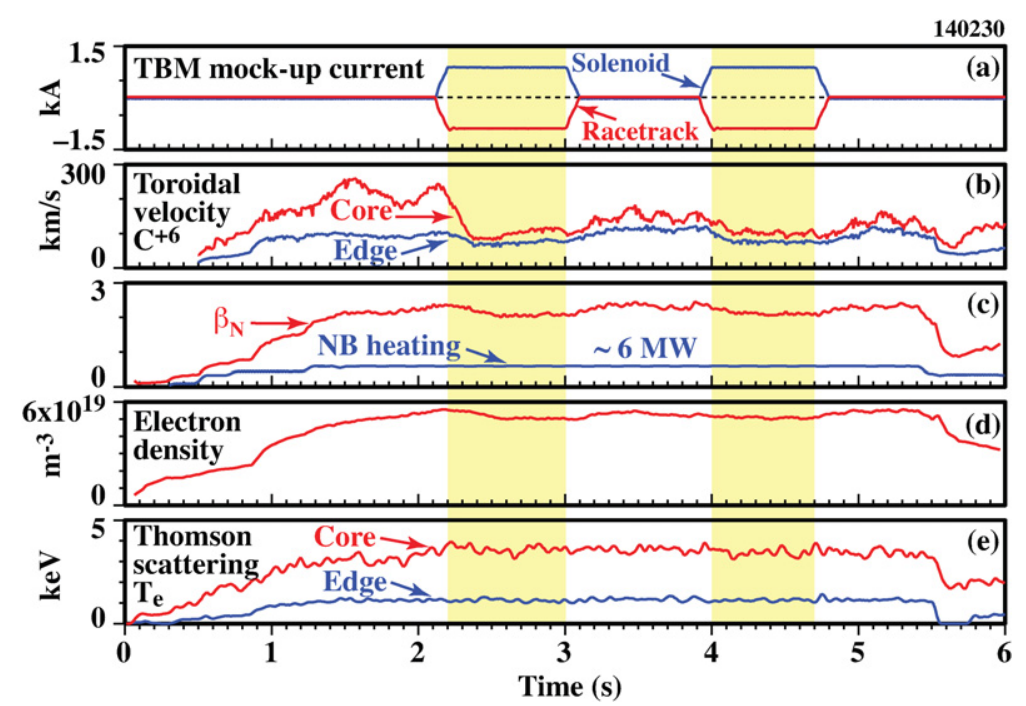

Figure 7. Time history of a representative TBM mock-up experimental pulse. (a) Mock-up racetrack and solenoid coil currents. (b) Toroidal velocity of $\mathrm{C}^{+6}$ ions in the plasma core and edge, from charge exchange recombination (CER) spectroscopy. (c) Normalized beta (read left scale) and neutral beam heating power (not to scale). $(d)$ Line-average electron density, from interferometer chord. (e) Electron temperature in plasma core and edge, from Thomson scattering data.

usually strongly dominated by the mock-up field. However, for consistency we always report results here as a function of the $\delta$ defined above.

In ITER the local ripple from the TBMs plus the corrected TF coil is expected to be $\delta \sim 1.2 \%$. The DIII-D mock-up was designed to reach $\delta>3 \%$ at full DIII-D toroidal field. In the experiments reported here, $\delta$ was varied by three methods. Most commonly we varied the current in the mockup coils. Secondly, we varied the plasma $R_{\text {midout }}$ to change the distance between the plasma boundary and the mock-up, and thus vary $\delta$. The minimum major radius $R_{\text {midin }}$ was kept constant while $R_{\text {midout }}$ was varied. This caused small changes to toroidal aspect ratio, triangularity, and other plasma parameters. Lastly, we varied the major radius position of the mock-up over a range equivalent to $0.5 \mathrm{~m}$ in ITER.

The time history of a representative TBM mock-up experimental shot, 140230, is shown in figure 7. Plasma 140230 is like 140149, shown in figure 6. It has a long ELMy $\mathrm{H}$-mode phase with steady $6 \mathrm{MW}$ of co-injected neutral beams. Two mock-up current pulses are applied at an amplitude that makes the combined local ripple $\delta=3 \%$. The second mockup pulse tests reproducibility and also the recovery of the plasma after the first mock-up pulse. Toroidal plasma flow, indicated approximately by $\mathrm{C}^{+6}$ ion toroidal velocity, decreases by $>50 \%$ in the plasma core during the first mock-up pulse. Although the velocity does not recover its original high value after the first pulse, both core and edge velocities equilibrate to about their same values during the second mock-up pulse as during the first. The line-average electron density $\bar{n}_{\mathrm{e}}$ and global $\beta_{\mathrm{N}}$ as well as total stored energy (not plotted) experience smaller decreases during the mock-up pulses. In contrast, the electron temperature $T_{\mathrm{e}}$ and deuterium ion temperature (not plotted) are not significantly affected.

\section{Results of TBM mock-up error field experiments}

In this section the results of the TBM mock-up experiments are presented and discussed by experimental topic.

\subsection{Negligible and small TBM mock-up effects}

Plasma startup: the combined local ripple of $2.7 \%$ had no measurable effect on either plasma initiation or on locking during the plasma current rampup, in an otherwise conventional DIII-D plasma startup at $B_{\mathrm{T}}=2.0 \mathrm{~T}$.

L-mode confinement: L-mode plasma confinement was only weakly affected by the mock-up. This is qualitatively consistent with the general weakening of confinement degradation by the mock-up error field with decreasing $\beta$.

$L-H$ power threshold: the $\mathrm{L}-\mathrm{H}$ transition power threshold was unaffected within experimental error by the mock-up field, tested up to $\delta=3.1 \%$ at $B_{\mathrm{T}}=1.7 \mathrm{~T}$. This absence of effect on the $\mathrm{L}-\mathrm{H}$ threshold was true for co-current neutral beam injection, which injects angular momentum into the plasma in the plasma current direction, as well as for torque-free balanced beam and electron cyclotron heating.

$H$-mode pedestal: the mock-up did not change the $\mathrm{H}$-mode pedestal significantly, except for density, which was reduced both globally and in the pedestal (density pumpout). The rigidity of the other temperature values and profiles is reminiscent of the response of the ELMy H-mode pedestal to $n=3$ RMPs applied for ELM suppression in DIII-D [12]. However, the mock-up field itself did not suppress ELMs in any of the experimental plasmas used.

ELM characteristics: the TBM mock-up field had no significant effect on ELM characteristics, except that a reduction in type I ELM frequency was seen in a plasma that was run close to an $\mathrm{H}$ - to L-mode back transition.

ELM control by RMPs: the mock-up had no significant effect on the ability of an $n=3$ RMP applied by the DIII-D I-coils to suppress type I ELMs [12].

Divertor power distribution: the plasma footprint on the divertor target varied little in response to the mock-up field. In particular, there was no sign of radial splitting of the footprint, such as is observed when resonant magnetic fields are applied.

Fast ion losses: detailed measurements indicated that incremental global loss of injected neutral beam fast ions due 
to the TBM mock-up field was small, no greater than the measurement error bars. This experimental result is consistent with previous calculations for ITER with TBMs using Monte Carlo fast ion codes [13]. Local heating of the plasma-facing tiles protecting the mock-up was observed, especially when the plasma-tile separation was reduced to $\sim 4 \mathrm{~cm}$, corresponding to $R_{\text {midout }}=2.33 \mathrm{~m}$. Even then, the local power loss was too small to be of global significance. Ongoing numerical analysis suggests that this particular enhanced tile heat load was due at least in part to prompt beam ion losses [14].

MHD activity: there were a few examples where the TBM mock-up field appeared to enhance the amplitude of MHD activity in the plasma. However, no consistent effect could be isolated, because most of the experimental H-mode plasmas already had irreproducible, time varying, non-linearly saturated neoclassical tearing mode (NTM) activity. NTM instabilities in these DIII-D plasmas are sensitive to small changes in the plasma radial profiles that were not well controlled. The fact that this activity was little affected by the mock-up perturbation is consistent with the absence of any effects on stability by a saturated full ferromagnetic wall in JFT-2M experiments [15, 16].

Poloidal magnetization: the presence or absence of the poloidal magnetization (solenoid) component of the mockup field had small, barely observable effects on plasma confinement. The larger toroidal magnetization (racetrack) current was unchanged during this experiment.

\subsection{Fast fusion product loss}

In these neutral beam heated DIII-D plasmas there was sufficient DD fusion that the product $1 \mathrm{MeV}$ tritons could be measured barely by the $14 \mathrm{MeV}$ DT fusion neutrons they made before escaping or slowing down. The gyroradius of a $1 \mathrm{MeV}$ triton in DIII-D is large, about $a / 3$, so an increased fast triton loss rate can be a sensitive indicator of perturbed $1 \mathrm{MeV}$ triton orbits. The triton generation and loss rates were calculated, respectively, from the measured $2.5 \mathrm{MeV}$ neutron rate and the DT $14 \mathrm{MeV}$ neutron rate, with the help of a time-dependent model similar to [17]. Figure 8 shows results from 14 similar DIII-D shots with mock-up pulses (combined to improve statistics). The best fit found by the model was $33 \pm 5 \%$ additional fast triton loss due to the mock-up pulse. A subsequent calculation of the same problem using the full orbit code SPIRAL [14], which is suitable for calculating very large ion orbits, also found $\approx 33 \%$ additional triton loss. The $3.5 \mathrm{MeV}$ fusion alpha orbits in ITER are smaller than the $1 \mathrm{MeV}$ triton orbits in DIII-D, relative to the respective plasma minor radii of the two tokamaks.

\subsection{Plasma rotation and $H$-mode confinement}

The largest observed effects of the TBM mock-up field were decreased locked mode tolerance, braking of plasma toroidal rotation and reduction in $\mathrm{H}$-mode confinement. They may be of concern to the IO because they affect fusion power gain, especially if their scalings from DIII-D to ITER are unfavourable. First, we present and discuss the experimental data. In section 4.4, we present evidence that these effects might be attenuated by a simple $n=1$ magnetic field applied to the plasma from error correction coils.

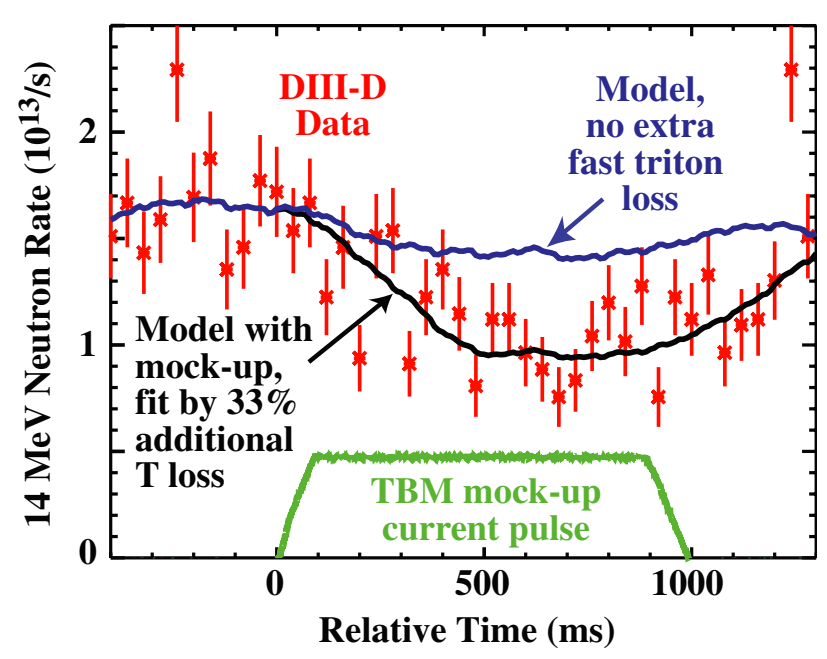

Figure 8. $14 \mathrm{MeV}$ DT neutron rate versus time relative to the start time of the TBM mock-up pulse. Data from 14 shots were combined to improve statistics. The top curve is the model calculation with triton loss rate unchanged from before the mock-up pulse. The shallow dip is due to reduced plasma density caused by the mock-up pulse. The black curve is calculated with triton loss rate increased $33 \%$ in the model during the mock-up current (best fit).

Reduction in the plasma toroidal velocity, $v_{\mathrm{T}}=\Omega R$, was the largest observed effect of the TBM mock-up perturbation experiments. Stable relative velocity reductions $-\Delta v_{\mathrm{T}} / v_{\mathrm{T} 0}$ up to $\sim 60 \%$ were observed at the highest local ripples $(\delta \sim 4 \%)$ in ELMing H-mode plasmas for the maximum available neutral beam injected torque/power ratio. Here $v_{\mathrm{T} 0}=\Omega_{0} R$ and $\Omega_{0}$ are the quasi-steady toroidal velocity and angular frequency just before application of the perturbation. Figure 9 shows such data for a variation of $\delta$ over a range from about 1 to 3 times the local ripple that is expected in front of a TBM port. In ELMy $\mathrm{H}$-mode plasmas, the relative velocity reductions were roughly 3 times greater than the corresponding relative reductions in the normalized energy confinement factor $H_{98}$. In these experimental scans, data were not taken for $\delta<15 \%$, where plasma response magnitudes were smaller than the data scatter.

Figure $10(a)$ shows that the relative responses of $\beta_{\mathrm{N}}, \bar{n}_{\mathrm{e}}$ and stored plasma energy $W$ to the mock-up field differed little from $H_{98}$ in figure 9. Since neither ion nor electron temperatures were much affected by the mock-up, the changes in $\beta_{\mathrm{N}}, W$ and $H_{98}$ appear to arise mainly from the decreased $\bar{n}_{\mathrm{e}}$. It is noteworthy that the proportionality factor $\sim 3$ between the response of $v_{\mathrm{T}}$ and the responses of $\bar{n}_{\mathrm{e}}, \beta_{\mathrm{N}}, W$, and $H_{98}$ to the mock-up field, is reminiscent of the toroidal angular momentum $L$ confinement degradations $\sim 2$ times greater than other confinement degradations documented in ITERlike hybrid scenario plasmas in DIII-D [18], suggesting that all these confinement reductions may be closely linked to, or even caused by, the changes in $v_{\mathrm{T}}$ and/or $L$. Figure $10(b)$ shows that the confinement degradations from the TBM mockup field increase also with $\beta_{N}$, in qualitative agreement with the general increasing sensitivity of tokamak plasma to error fields with $\beta$. In these experimental scans, data were not taken for $\beta_{\mathrm{N}}<2$ where plasma response magnitudes were smaller than the data scatter.

When the mock-up was moved radially outwards, away from the plasma, the confinement losses decreased less rapidly 


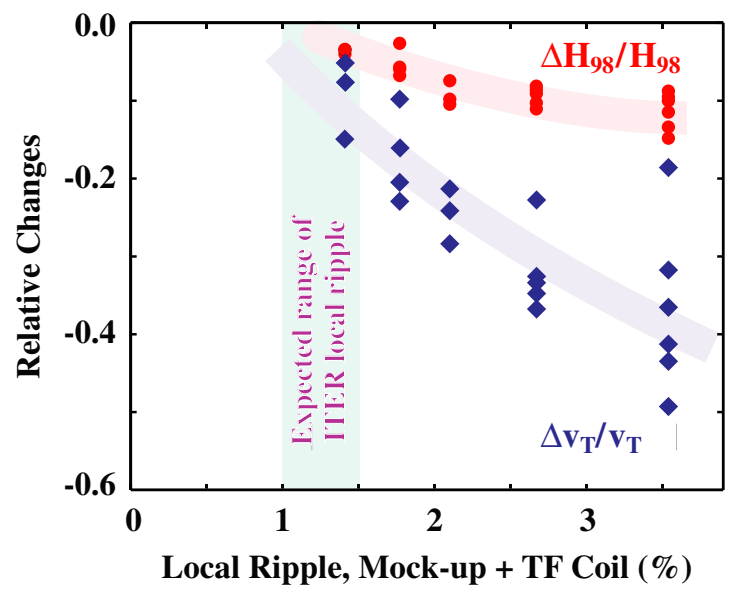

Figure 9. Relative changes in $v_{\mathrm{T}}$ and $H_{98}$ as a function of local ripple $\delta$ as the mock-up coil current was varied. This parameter scan was performed with the shot 140149 parameters, except $R_{\text {midout }}=2.32 \mathrm{~m}$ and $\beta_{\mathrm{N}} \approx 2.1-2.4$. The IO goal for $\delta$ in front of one ITER TBM port is $\approx 1.2 \%$.
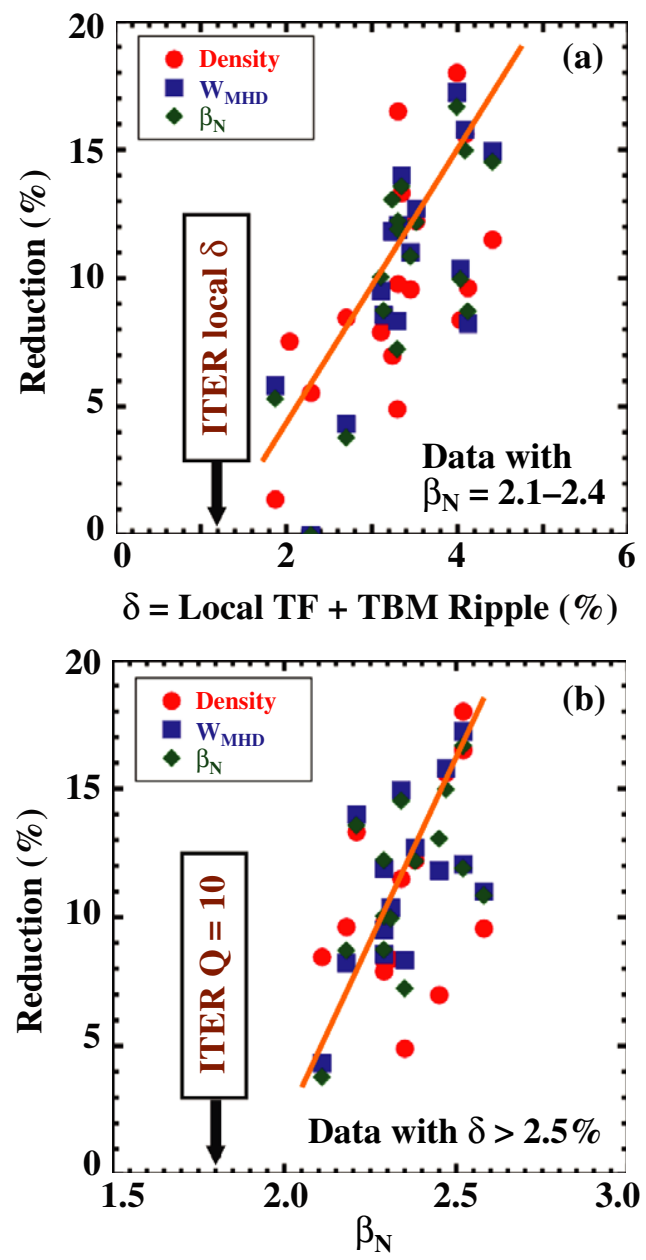

Figure 10. Relative reductions in density, stored energy $W_{\mathrm{MHD}}$, and $\beta_{\mathrm{N}}$ for ELMy H-mode plasmas, due to the mock-up field, $(a)$ as a function of $\delta$ within a restricted range of $\beta_{\mathrm{N}}$, and $(b)$ as a function of $\beta_{\mathrm{N}}$ for cases with $\delta>2.5 \%$. All plasmas had $R_{\text {midout }}=2.30 \mathrm{~m}$ and $q_{95}=3.5$.

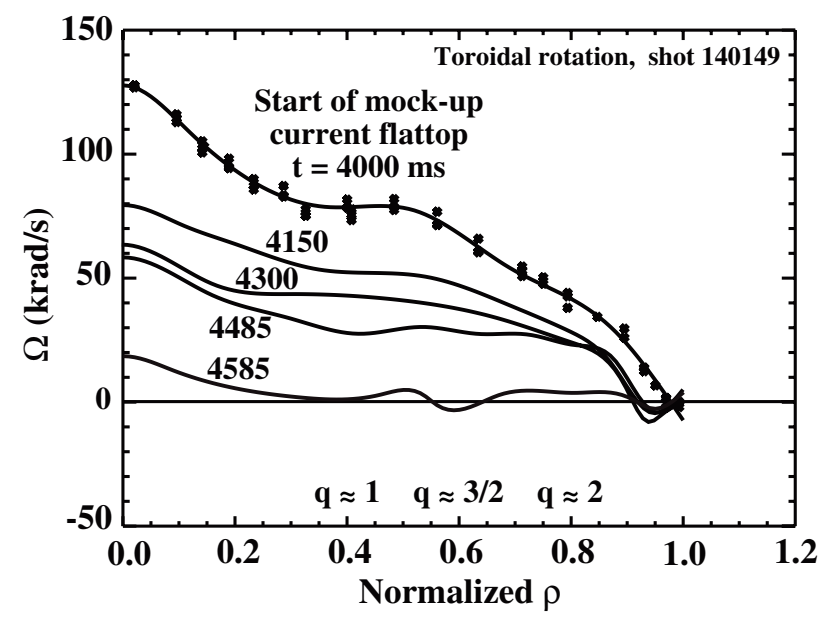

Figure 11. Evolution of plasma $\mathrm{C}^{+6}$ rotation profiles with time, measured by CER spectroscopy, in shot 140149 , from the start of the second mock-up current pulse flattop at $t \approx 4000 \mathrm{~ms}$. Minor radius coordinate in this figure is $\rho=$ sqrt (normalized toroidal magnetic flux). Three time-sequential data points from each CER view were used to produce the splined curves. Data points are shown for the first time to indicate the data scatter.

with the corresponding local ripple change than when the change was made by varying mock-up coil currents. Perhaps this weaker dependence arises from the increasing spatial extent of the TBM field at the plasma as the distance between the two is increased. Regardless of the cause, this result signifies that the confinement degradation is not just a function of $\delta$ alone.

ELMing H-mode plasma rotation was occasionally locked by a strong mock-up field at sufficiently unfavourable combinations of high $\beta_{\mathrm{N}}$, low $q$ and low rotation. Figure 11 shows the history of a braking event that eventually led to plasma rotation locking during the second mock-up pulse in shot 140149, shown at an earlier time in figure 6. This shot's history proceeded much like shot 140230 , shown in figure 7 . However, $\beta_{\mathrm{N}}$ not only recovered after the first mock-up pulse, but it grew to 2.7 , larger than in 140230 . Referring to figure 11 , at $t=4000 \mathrm{~ms}$ the mock-up current pulse had just reached its programmed value for a local ripple $\delta \approx 30 \%$. The rotation profile $\Omega_{0}(\rho)$ at this time is typical. For a while, rotation decreased at a moderate rate across the full profile. Such braking, that is not spatially concentrated about a low-rational$q$ magnetic surface, is typical of non-resonant braking [19]. The observation of braking throughout the plasma, even to the magnetic axis, is suggestive of braking by low- $n$ non-resonant modes able to penetrate the plasma deeply. Furthermore, the early and persistent braking and flow direction reversal at $\rho>0.9$ is suggestive of a non-resonant, neoclassical toroidal viscous (NTV) offset torque generated $[20,21]$ by the very localized, high $m$ and $n$ spatial harmonics of the TBM-like magnetic field, noted earlier. Similar flow reversal from strong toroidal field ripple was reported near the edges of the JT-60U [3] and JET [4] tokamaks. By $t \approx 4300 \mathrm{~ms}$ the decay had slowed to almost a stationary profile that was about $0.5 \Omega_{0}(\rho)$ across most of the profile. However, the toroidal flow was never quite in steady-state torque balance, and as typical for toroidal braking by non-axisymmetric magnetic fields, the torque balance bifurcated $[22,23]$ to one dominated by strong 

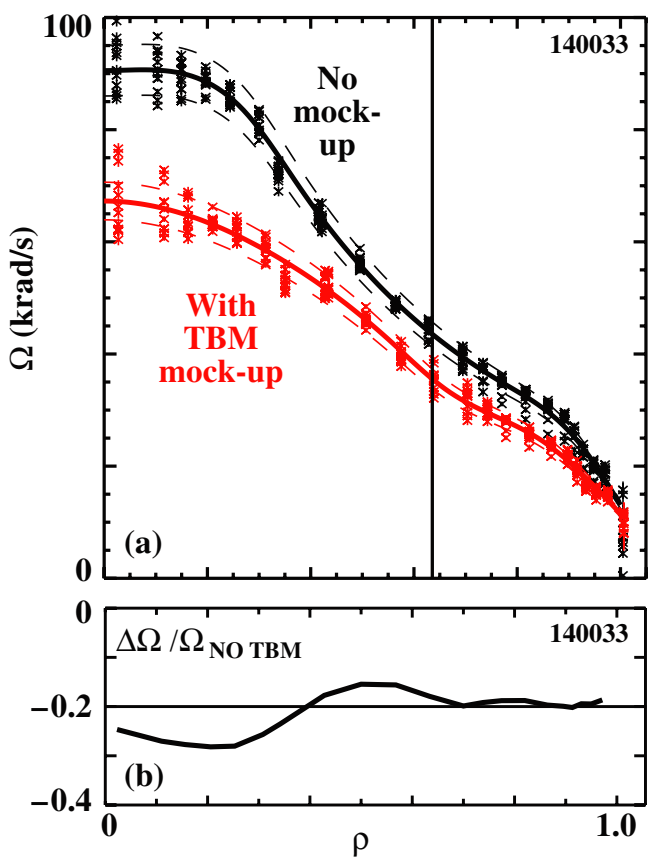

Figure 12. Radial profiles of toroidal rotation of $\mathrm{C}^{+6}$ measured by CER spectroscopy, in shot 140033, (a) without and with the TBM mock-up field $(\delta \approx 3.3 \%)$. (b) The normalized difference between the two profiles in $(a)$. The vertical line at $\rho \approx 0.63$, corresponding to $R \approx 2.12 \mathrm{~m}$, marks the location of the CER view used to make figure 13 .

resonant electromagnetic braking. This process started a bit before $t=4485 \mathrm{~ms}$, and it terminated in a steady locked state, which was firmly established by $t=4585 \mathrm{~ms}$. In this final state the locking $(\Omega \approx 0)$ appears to be strongest near the $q \approx 1$ and $q \approx 3 / 2$ rational surfaces at $\rho \approx 0.4$ and 0.6 , respectively. It is weak near the $q=2$ surface at $\rho \approx 0.8$. Braking localized in the vicinity of low-rational surfaces is a characteristic of strong resonant electromagnetic braking, due to local resistive and viscous dissipation where the moving plasma screens the resonant components of a non-axisymmetric magnetic field.

Rotation braking in ELMy $\mathrm{H}$-mode plasmas was investigated in additional detail. These experiments were conducted at higher $q_{95}(\approx 4.0)$ and lower $\beta_{\mathrm{N}}(\approx 1.5)$, in order to have less shot-to-shot variation than the plasmas presented so far in this paper. Neutral beam heating was used, and the ratio between co- and counter-injected beams was employed to control the injected torque as an experimental variable while maintaining constant $\beta_{\mathrm{N}}$. Figure 12(a) shows the profiles of the toroidal rotation in shot 140033 , without and with the TBM mock-up field applied to make $\delta \approx 3.3 \%$. Although the mock-up vacuum magnetic fields decay rapidly into the plasma, the observed relative reductions $-\Delta \Omega / \Omega_{0}$ extended all the way to the magnetic axis as in figure 11. Figure $12(b)$ shows that the ratio $-\Delta \Omega / \Omega_{0}$ was approximately uniform across the plasma radius. The absence of local braking at integer- $q$ magnetic surfaces again indicates that there was no strong resonant magnetic braking, a conclusion qualitatively consistent with the smallness of the numerically computed $n=1$ resonant harmonic content of the TBM perturbation. Furthermore, figure 13 shows that a linear relation, $\Delta \Omega \approx$ $-f \Omega_{0}$, appears to hold as well when the NBI torque is varied

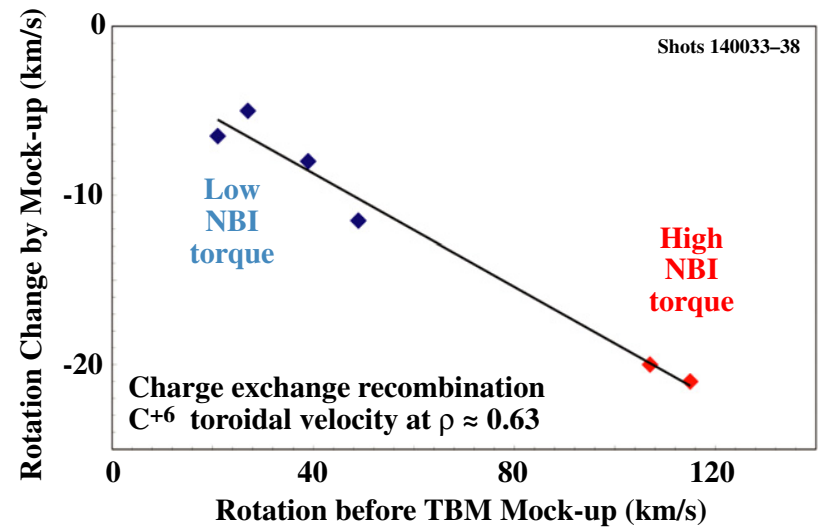

Figure 13. Change in toroidal velocity at $\rho=0.63$ in shots similar to 140033 and with the same $\delta$, while the pre-mock-up velocity $v_{\mathrm{T} 0}$ was changed by varying the NBI torque.

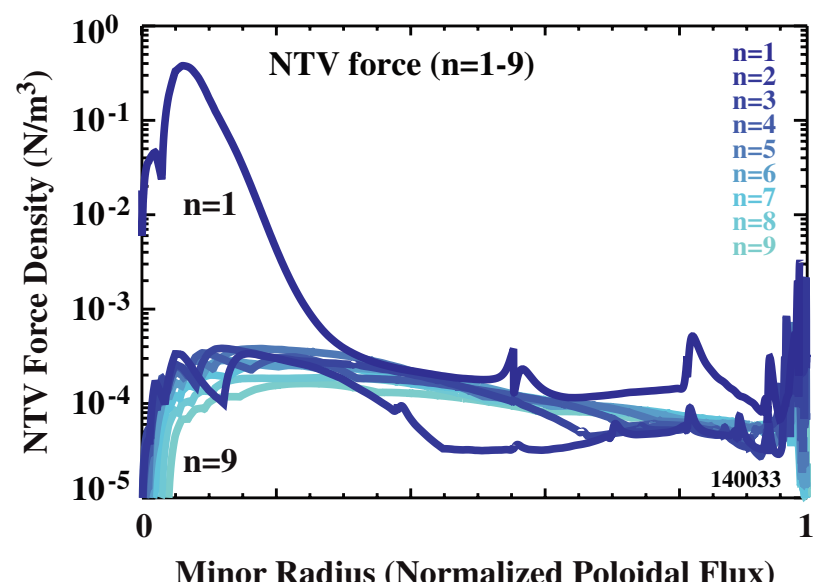

Figure 14. Radial profiles of the NTV braking force density by the mock-up field, calculated by IPEC for shot 140033. The curve for each toroidal harmonic number $n$ is the force density summed over all the helical harmonics having that $n$.

among similar plasmas with the same local ripple applied. Here $f$ is a scalar factor independent of torque but dependent on the local ripple and $\beta$. Linear dependence of $\Delta \Omega$ on the initial rotation rate is a property of non-resonant braking, such as neoclassical toroidal viscosity braking. NTV braking has been identified in previous experiments in NSTX [24] and DIII-D [19].

Theoretical NTV braking by the mock-up field was evaluated numerically for shot 140033 presented in figure 12 , using the code IPEC [25] to compute the magnetic field within the plasma using a linear ideal MHD model, from which the NTV braking torque ([26] and references therein) was evaluated approximately. A realistic representation of the complicated mock-up $B$ field was applied to the plasma. Radial profiles of the computed NTV force density, due to the mockup helical Fourier harmonics from $n=1$ to $n=9$, are shown in figure 14. IPEC analysis identified plasma amplification of the $m, n=1,1$ ideal internal kink mode, which peaks on axis, as the main cause of the deep-core braking apparent in the figure. The computed global NTV torque was, however, $\sim 3$ times larger than the global braking torque inferred from a balance between the experimental injected torque and 
rotation slowing. Also, the contrast between the computed central peak and the rest of the NTV force distribution is too large for consistency with the experimental profiles in figure 12. There are several known limitations to the IPEC NTV calculation. Firstly, the computed $n=1$ amplification depends very sensitively on $q(0)$ when $q(0) \sim 1$, which is the case in this plasma. Furthermore, the computed NTV force density increases quadratically with the computed local non-axisymmetric magnetic field. Thus, if the actual $n=1$ amplification was smaller by a factor of 2 than that computed by IPEC, the $n=1 \mathrm{NTV}$ force density peak in the figure would be smaller by a factor of 4 , which would yield closer agreement with the experimental global braking. Secondly, the $n>1$ harmonic contributions in figure 14 act almost uniformly throughout the plasma. Although none of these higher harmonics contributes as much force density individually as the $n=1$ peak, there are many of them, and the sum of all their contributions is not negligible. However, there is almost no reduction of the braking contributions from the $n=2$ through $n=9$ harmonics shown in the figure. In fact, significant harmonic families up to $n \sim 30$ are present in the computed mock-up field, and they should be included in future numerical braking analysis. Thirdly, the NTV theory [20,27,28] is complicated and is still being validated against experiment. Finally, there are other contributions to braking torque, such as turbulence. The semi-quantitative correspondence between the numerical modelling and experiment should be treated with caution. However, in the next section we present additional experimental evidence that $n=1$ harmonics are the most important.

\subsection{Locking of plasma rotation}

Rotation locking by the TBM mock-up field was studied in dedicated L- and H-mode plasmas. In both modes the mockup field decreased plasma tolerance to locking to a controlled known $n=1$ 'proxy error' test field. (Proxy error is a known field applied to simulate an error field.)

The L-mode study made use of the same highly reproducible low-density, ohmically heated plasmas that are used for many locking experiments and to develop low- $\beta$ error field corrections at DIII-D [29]. These plasmas are especially sensitive to the few $n=1$ error harmonics that couple to a weakly stable plasma external kink mode, and they lock reproducibly at a critical line-average electron density $\bar{n}_{\text {crit }}$ that is very nearly linearly proportional to the strength of a selected $n=1$ proxy error field. The lower the density to which the plasma survives until a locked mode appears, the smaller the dangerous component of the total error field acting on the plasma. Similar plasmas are used at other tokamaks, which facilitates inter-machine comparison of error field locking experiments. The low- $\beta$ test plasma used to study locking by the mock-up field had a pumped doublenull diverted configuration and was run at $q_{95} \approx 3.3$. Its critical line-average electron density for locking to the DIII-D intrinsic machine error was $\bar{n}_{\text {crit }} \approx 0.85 \times 10^{19} \mathrm{~m}^{-3}$. When the standard $n=1$ DIII-D correction field was applied by the error correcting I-coil array to correct the intrinsic error, $\bar{n}_{\text {crit }}$ was almost halved, to $\bar{n}_{\text {crit }} \approx 0.43 \times 10^{19} \mathrm{~m}^{-3}$. These are within experimental error of historical values. In figure 15

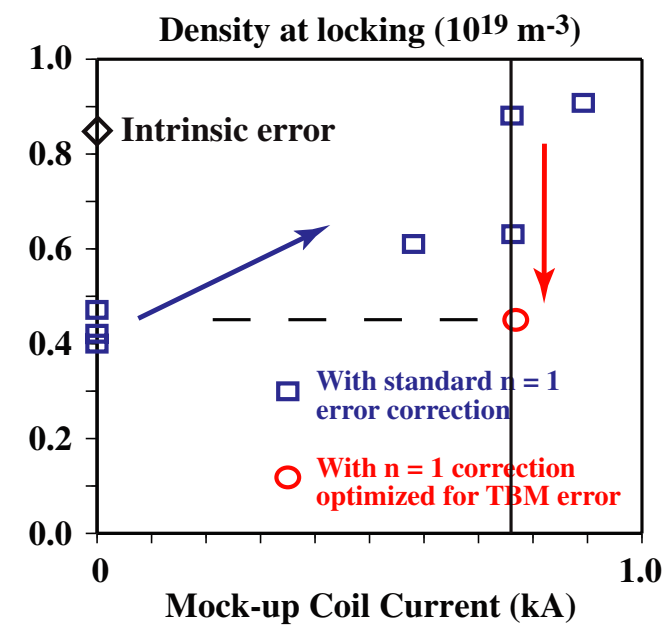

Figure 15. Critical density of locking in ohmic test plasmas as a function of mock-up racetrack coil current. One $\bar{n}_{\text {crit }}$ datum for the uncorrected machine intrinsic error, and four data taken with standard error correction active, appear on the left axis at zero mock-up current. The slanted arrow points to data for three mock-up currents (squares) and still standard error correction. The circle marks $\bar{n}_{\text {crit }}$ after the $n=1$ error correction was reoptimized while the mock-up error was present. The vertical line at $0.76 \mathrm{kA}$ indicates $\delta=2.2 \%$.

the intrinsic and corrected experimental $\bar{n}_{\text {crit }}$ data for zero mock-up current appear along the left vertical axis. With the standard error correction still active, the addition of a mockup local ripple of $\delta=2.2 \%$ (racetrack current $\approx 0.76 \mathrm{kA}$ ) approximately doubled the critical density for locked mode formation to $\bar{n}_{\text {crit }} \approx 0.89 \times 10^{19} \mathrm{~m}^{-3}$ to about the same $\bar{n}_{\text {crit }}$ as for the uncorrected intrinsic error. Thus, the mock-up field significantly increased sensitivity to locking. Next, the $n=1$ error compensation from the I-coil array was reoptimized experimentally for the intrinsic plus mock-up errors together, and $\bar{n}_{\text {crit }}$ was reduced to $0.46 \times 10^{19} \mathrm{~m}^{-3}$, the red circle in figure 15 . To within experimental error this is equal to $\bar{n}_{\text {crit }}$ for the corrected intrinsic error alone. In this experiment, at least, the additional locked mode sensitivity induced by the TBM mock-up error was eliminated.

The experimental low- $\beta$ locking threshold increase by the mock-up field and its compensation by the I-coil connected array in its $n=1$ error field correction configuration were both matched semi-quantitatively by IPEC calculations. This correspondence between experiment and theory supports the case for the important role of error field components that couple to the dominant $n=1$ ideal kink mode [26], even for the extremely spatially localized TBM mock-up error field that has $\sim 100$ times more $n>1$ than $n=1$ harmonic energy.

The controlled H-mode locking experiments were performed in ISS plasma of figure 6 , except that $I_{\mathrm{p}}$ was reduced to make $q_{95} \approx 4.1$ for better reproducibility. Combined co- and counter-injected neutral beams were used to vary the net NBI torque while keeping $\beta_{\mathrm{N}} \approx 2.1$. Tolerance against locking was quantified as the minimum $n=1$ proxy error, applied by the well-characterized DIII-D I-coil array, needed to produce a locked mode. Tolerance against locking was decreased by increased TBM mock-up field and/or decreased injected torque. The experiments showed that the critical proxy error amplitudes to cause locking depended only on 
the plasma rotation existing before the programmed proxy error field ramp began. The critical field was independent of the means employed to reduce the rotation. This result indicates that the TBM mock-up reduces the H-mode error field tolerance by exerting its own torque on the plasma.

Since locked modes caused by the mock-up were not problematic in the initial experimental survey, locked mode topics had been ranked at a relatively low priority for subsequent detailed experiments. As a result, there was insufficient experimental time to test whether the I-coil $n=1$ error compensation could help avoid locked modes in $\mathrm{H}$-mode plasmas as it did in the ohmic L-mode plasmas. This experiment remains as future work.

\section{Discussion}

ITER will have TBMs containing ferromagnetic steel in three of its midplane ports, separated far enough that their magnetic fields at the plasma are spatially localized and isolated from one another. The DIII-D experiments used a purpose-built TBM error field mock-up coil set placed in one midplane port to investigate possible effects on the plasma of ITER TBMs. To compensate the unequal number of error sources, the local ripple $\delta_{\text {DIII-D }}$ in the DIII-D experiments was usually made $\sim 3$ times as large as the local ripple $\delta_{\text {ITER }}$ expected in front of one ITER TBM.

Many effects were insignificant in DIII-D and perhaps they will remain so in ITER. These include effects on plasma startup, L-mode confinement, L-H transition power threshold, H-mode pedestal parameters (except for pedestal density, which was decreased), ELM behaviour, including ELM control by RMPs and MHD activity. However, other effects may be large enough to be of concern for ITER, depending on how they extrapolate. These include braking of plasma toroidal rotation, reduction in $\mathrm{H}$-mode confinement and reduced tolerance against locked modes.

At present it is not well understood how to confidently extrapolate from the DIII-D experiments and predict the consequences for ITER. We illustrate the matter by a simple example. Consider the case where the amplitude $A$ of some TBM effect due to the perturbation from one port, whether in ITER or DIII-D, scales with the local ripple amplitude to some power $\alpha$, that is

$$
A \sim \delta^{\alpha} .
$$

Let there be $N_{\text {ITER }}$ identical well separated perturbing ports in ITER and assume that they contribute independently to $A_{\text {ITER }}$. Then $A_{\text {ITER }} \sim N_{\text {ITER }} \delta_{\text {ITER }}^{\alpha}$. However, $A_{\text {DIII-D }} \sim \delta_{\text {DIII-D }}^{\alpha}$ because there is only one DIII-D TBM error port. The scaling ratio $S$ from DIII-D to ITER would then be

$$
S=\frac{A_{\text {ITER }}}{A_{\text {DIII-D }}}=\frac{N_{\text {ITER }} \delta_{\text {ITER }}^{\alpha}}{\delta_{\text {DIII-D }}^{\alpha}}=N_{\text {ITER }}\left(\frac{\delta_{\text {ITER }}}{\delta_{\text {DIII-D }}}\right)^{\alpha} .
$$

For this discussion, let us now specialize to the mock-up experiments, where the most strongly perturbed shots had $\delta_{\text {DIII-D }} \approx N_{\text {ITER }} \delta_{\text {ITER }}$. For this condition it follows that $S=1 / N_{\text {ITER }}^{\alpha-1}$. If $\alpha=1$, then $S=1$; that is, the same amplitude $A$ of TBM effect would be observed in ITER as in DIII-D, regardless of the number of perturbation ports in ITER. However, if $\alpha=2$, then $S=1 / N_{\text {ITER }}$, in which case the level

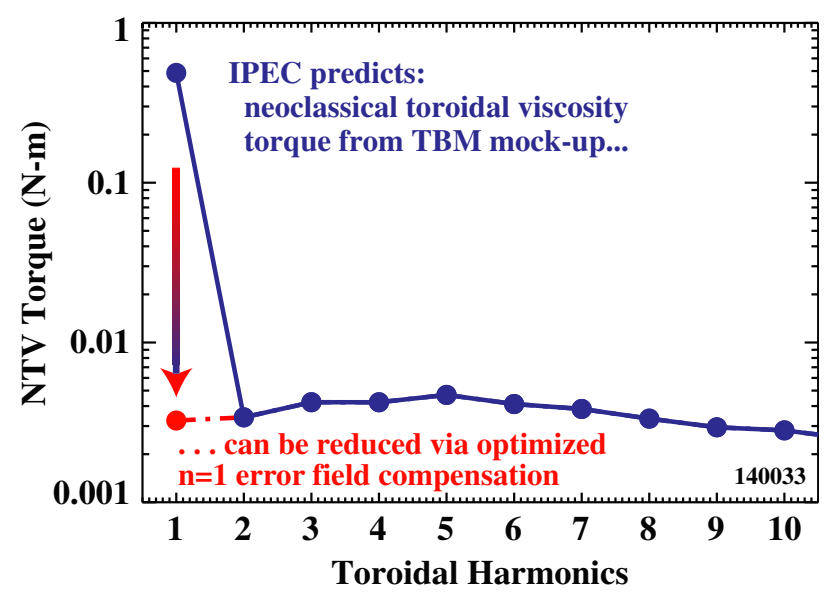

Figure 16. IPEC prediction of how the DIII-D I-coil might reduce the $n=1 \mathrm{NTV}$ torque to a negligible fraction of the total NTV torque. Each circle is the full-plasma torque generated by all the helical harmonics associated with the labelled number of toroidal periods, $n$.

of $A$ in ITER would be $N_{\text {ITER }}=3$ times smaller than amplitude of that effect in DIII-D. The case $\alpha=2$ is not so implausible, since plasma braking by magnetic perturbations is theoretically proportional to $\delta^{2}$. One might argue that the relative reductions in density, stored energy and $\beta_{\mathrm{N}}$ in figure $10(a)$ could be read as having a $\delta^{2}$ dependence, but this cannot be distinguished from an offset linear dependence with the available data. Future experiments should be developed to produce better data at small $\delta$ and possibly establish a firmer basis for extrapolation to ITER. Of course, TBM effects on the plasma might depend on more variables than just $\delta^{\alpha}$. A rigorous theoretical analysis is needed.

The surprising outcome of this TBM error investigation is that those effects that were large enough in DIII-D to present some concern for ITER might also be greatly reduced in magnitude by the simple expedient of good compensation of the few $n=1$ most critical helical harmonics. The most dangerous harmonics appear to be amplified internally by the tokamak plasma and to then cause significant braking of plasma rotation. Successful TBM error compensation in H-mode would facilitate sustained plasma rotation at ITERrelevant $\beta_{\mathrm{N}}$, which might benefit plasma confinement and stability, and especially locked mode avoidance. Numerical analysis with IPEC of the mock-up error braking and locking experiments reported here gives some credence to this scenario. The IPEC numerical study of NTV braking, summarized in figure 14, was extended to determine whether the I-coil $n=1$ correction field could be adjusted to reduce the large $n=1$ NTV braking. The optimum numerical result, in figure 16, reduced the $n=1$ contribution to the plasma NTV braking torque from dominance, as in figure 14 , to about the same as the contributions from the other harmonics shown. The same caveats, given at the end of section 4.3 about the uncertain accuracy of the IPEC-NTV model and calculations at the present state of the art, also apply to this prediction for $\mathrm{H}$-mode tolerance to locked modes. Future experiments should test $n=1$ compensation of the TBM error for ELMy H-mode plasmas. This should be done not only for plasmas having strong toroidal driving torque from neutral beam injection in 
the plasma current direction (co-injection), but it should also be extended to plasmas having reduced torque injection and for ECH-heated plasmas.

\section{Acknowledgment}

This work was supported in part by the US Department of Energy under DE-FC02-04ER54698, DE-AC52-07NA27344, DE-AC02-09CH11466, SC-G903402, DE-FG02-04ER54761, DE-AC05-00OR22725, DE-FG02-07ER54917 and DEFG02-08ER54984. The views and opinions expressed herein do not necessarily reflect those of the ITER Organization.

\section{References}

[1] Giancarli L. et al 2010 Fusion Eng. Des. 851829

[2] Amoskov A. et al 2008 Magnetic field produced by test blanket modules, ITER_D_2FB3NX (Nov 28)

[3] Urano H. et al 2007 Nucl. Fusion 47706

[4] de Vries P.C. et al 2008 Nucl. Fusion 48035007

[5] Lackner K. 2007 private communication, Max-Planck-Institut für Plasmaphysick, EURATOM Association, 85748 Garching, Germany

[6] Snipes J.A. et al 2010 Results of ITER test blanket module mock-up experiments on DIII-D Proc. 37th EPS Conf. on Plasma Physics (Dublin, Ireland, 21-25 June 2010) vol 34A (ECA) P1.1093 and http://ocs.ciemat.es/ EPS2010PAP/html/author.html

[7] Schaffer M.J. et al 2010 Proc. 23rd Int. Conf. on Fusion Energy 2010 (Daejeon, Korea, 2010) (Vienna: IAEA) CD-ROM file ITR/1-3 and http://www-naweb.iaea.org/ napc/physics/FEC/FEC2010/html/index.htm
[8] Schaffer M.J. 2010 Bull. Am. Phys. Soc. 55 BI3.00001

[9] Pustovitov V.D. 2009 Phys. Plasmas 16052503

[10] Jackson G.L. 2003 Initial results from the new internal magnetic field coils for resistive wall mode stabilization in the DIII-D tokamak Proc. 30th EPS Conf. on Plasma Physics (St Petersburg, Russia, 7-11 July 2003) vol 27A (ECA) P-4.47 http://epsppd.epfl.ch/ StPetersburg/html/aidx0.html

[11] Strait E.J. et al 2004 Phys. Plasmas 112505

[12] Evans T.E. et al 2008 Nucl. Fusion 48024002

[13] Shinohara K. et al 2009 Fusion Eng. Des. 8424

[14] Kramer G. et al 2011 Fast ion effects during test blanket module simulation experiments in DIII-D Nucl. Fusion 51 at press

[15] Tsuzuki K. et al 2003 Nucl. Fusion 431288

[16] Tsuzuki K. et al 2006 Nucl. Fusion 46966

[17] Duong H.H. and Heidbrink W.W. 1993 Nucl. Fusion 33211

[18] Politzer P.A. et al 2008 Nucl. Fusion 48075001

[19] Reimerdes H. et al 2009 Nucl. Fusion 49115001

[20] Cole A.J., Hegna C.C. and Callen J.D. 2007 Phys. Rev. Lett. 99065001

[21] Garofalo A.M. et al 2008 Phys. Rev. Lett. 101195005

[22] Jensen T.H., Leonard A.W. and Hyatt A.W. 1993 Phys. Fluids B 51239

[23] Fitzpatrick R. 1998 Phys. Plasmas 53325

[24] Zhu W. et al 2006 Phys. Rev. Lett. 96225002

[25] Park J.K., Boozer A.H. and Glasser A.H. 2007 Phys. Plasmas 14052110

[26] Park J.K. et al 2009 Phys. Plasmas 16056115

[27] Shaing K.C., Sabbagh S.A. and Chu M.S. 2010 Nucl. Fusion 50025022

[28] Park J.K. et al 2009 Phys. Rev. Lett. 102065002

[29] Scoville J.T. and La Haye R.J. 2003 Nucl. Fusion 43250 